\title{
OPEN SPARC promotes insulin secretion through down-regulation of RGS4 protein in pancreatic $\beta$ cells
}

\author{
$\mathrm{Li} \mathrm{Hu}^{1,2,3}$, Fengli He ${ }^{1,2,3}$, Meifeng Huang ${ }^{1,2,3}$, Oian Zhao ${ }^{4}$, Lamei Cheng ${ }^{4}$, Neveen Said ${ }^{5}$, \\ Zhiguang Zhou ${ }^{1,2,3}$, Feng Liu ${ }^{1,2,3,6}$ \& Yan-Shan Dai ${ }^{1,2,3,7 凶}$
}

SPARC-deficient mice have been shown to exhibit impaired glucose tolerance and insulin secretion, but the underlying mechanism remains unknown. Here, we showed that SPARC enhanced the promoting effect of Muscarinic receptor agonist oxotremorine-M on insulin secretion in cultured mouse islets. Overexpression of SPARC down-regulated RGS4, a negative regulator of $\beta$-cell M3 muscarinic receptors. Conversely, knockdown of SPARC up-regulated RGS4 in Min6 cells. RGS4 was up-regulated in islets from sparc-I- mice, which correlated with decreased glucose-stimulated insulin secretion (GSIS). Furthermore, inhibition of RGS4 restored GSIS in the islets from sparc -/- mice, and knockdown of RGS4 partially decreased the promoting effect of SPARC on oxotremorine-M-stimulated insulin secretion. Phosphoinositide 3-kinase (PI3K) inhibitor LY-294002 abolished SPARC-induced down-regulation of RGS4. Taken together, our data revealed that SPARC promoted GSIS by inhibiting RGS4 in pancreatic $\beta$ cells.

The etiology of Diabetes involves both insulin resistance and decreased insulin secretion in $\beta$ cells ${ }^{1-3}$. Adipose tissue inflammation plays an important role in the development of obesity-induced insulin resistance ${ }^{2,4}$. In the prediabetic insulin-resistant state, islets respond to the increased insulin demand by increasing insulin secretion and $\beta$-cell mass, which generates compensatory hyperinsulinemia and maintains relative normal glycaemia. When insulin secretion is insufficient to compensate for the insulin resistance, type 2 diabetes emerges. The early $\beta$-cell dysfunction is manifested as impaired glucose-stimulated insulin secretion (GSIS), and loss of first phase GSIS can be detected in the earliest stages of type 2 diabetes ${ }^{1,3}$.

SPARC (Secreted Protein Acidic and Rich in Cysteine) is expressed mainly in bone and adipocytes, and it is a secreted extracellular matrix protein that is produced as a $32-\mathrm{kDa}$ matricellular glycoprotein ${ }^{5-9}$. SPARC mediates interactions between cells and their extracellular matrix, and targeted deletion of sparc results in compromised extracellular matrix in mice ${ }^{7,10}$. SPARC can bind to integrin beta 3 and activate integrin linked kinase ${ }^{11}$. Secretion of SPARC by adipose tissue is increased by insulin and leptin ${ }^{12,13}$. Adipose tissue is a major source of SPARC, which in turn inhibits adipogenesis and induces insulin resistance in adipose tissue in an autocrine or paracrine fashion ${ }^{14-16}$. SPARC has been shown to be involved in oxidative stress, neurogenesis, insulin resistance, glucose metabolism and Glut4 expression ${ }^{16-20}$.

SPARC is expressed in the stromal cells of mouse primary islets that can be detected by Western blot, and plays a role in reducing IGF-1-induced islet survival ${ }^{21}$. sparc-/- mice on high-fat diet showed an impaired insulin secretion capacity and a dramatically reduced expression of glucose transporter 2 in the islets ${ }^{22}$. SPARCdeficient mice exhibited impaired glucose tolerance ${ }^{22,23}$, and one of the underlying mechanism is reduced insulin secretion $^{22}$

Acetylcholine, which is released from intrapancreatic nerve endings, stimulates insulin secretion through binding and activation of M3 muscarinic receptor, resulting in increased intracellular calcium concentration in $\beta$ cells during food ingestion ${ }^{24}$. Mice selectively lacking M3 muscarinic receptor in pancreatic $\beta$ cells displayed impaired glucose tolerance and a dramatic reduction in insulin secretion, demonstrating that M3 muscarinic

\footnotetext{
${ }^{1}$ Department of Metabolism and Endocrinology, The Second Xiangya Hospital, Central South University, Changsha, Hunan, China. ${ }^{2}$ National Clinical Research Center for Metabolic Disease, The Second Xiangya Hospital, Central South University, Changsha, Hunan, China. ${ }^{3}$ Metabolic Syndrome Research Center, The Second Xiangya Hospital, Central South University, Changsha, Hunan, China. ${ }^{4}$ nstitute of Reproductive and Stem Cell Engineering, Central South University, Changsha, Hunan, China. ${ }^{5}$ Department of Cancer Biology, Wake Forest University School of Medicine, Winston-Salem, NC, USA. ${ }^{6}$ Department of Pharmacology, University of Texas Health Science Center, San Antonio, TX, USA. ${ }^{7}$ Present address: Bristol-Myers Squibb Company, Princeton, NJ, USA. ${ }^{\square}$ email: Ydai88@ csu.edu.cn
} 
receptor in $\beta$-cells is essential for maintaining normal glucose homeostasis ${ }^{24,25}$. RGS4 is a negative regulator of G protein-coupled M3-muscarinic receptor. Pancreatic $\beta$ cell-specific knockout of Rgs 4 did not alter basal glucose levels, but increased plasma insulin levels and muscarinic agonist-stimulated insulin release ${ }^{26}$, suggesting that selectively enhancing the M3 muscarinic receptor signaling in pancreatic $\beta$ cells could lead to novel treatment for patients with type 2 diabetes ${ }^{26}$.

To elucidate the mechanism by which SPARC regulates insulin secretion and $\beta$-cell physiology, we had analyzed insulin secretion in SPARC-deficient $\beta$ cells. Our results demonstrate that SPARC was essential for normal $\beta$-cell function and promotes insulin secretion by inhibiting RGS4 expression.

\section{Materials and methods}

Materials. sparc -/- mice were a gift from Dr. Neveen Said at University of Virginia, Charlottesville, Virginia, USA ${ }^{27}$ and were maintained in the accredited pathogen-free Second Xiangya hospital mice facility on a $12 \mathrm{~h}$ light/dark cycle ${ }^{28}$. C57BL/6 mice were purchased from Model Animal Research Center of Nanjing University. All experiments were approved by the Animal Care Research Committee of Second Xiangya Hospital (ACRCSXH) and carried out as per ACRCSXH guidelines.

Human sparc cDNA clone was described by us before ${ }^{29}$ and subcloned into pShuttle vector (Clontech). Adenovirus expressing human SPARC was constructed using Adeno-X expression system (Clontech) as described before $^{30,31}$. GAPDH, Horseradish peroxidase labeled donkey anti rabbit or donkey anti mouse antibodies were from Cell Signaling (Beverly, MA). Recombinant mouse SPARC protein (cat. number 942-SP-050) was purchased from R \& D systems. Oxotremorine M (Oxo-M) and LY294002 were purchased from Sigma-Aldrich. CCG4986 was purchased from ChemBridge (San Diego, CA).

Methods. Cell culture. Min6 cells were originally purchased from ATCC and were cultured in DMEM containing 15\% FBS, $25 \mathrm{mM}$ Glucose and $50 \mu \mathrm{M} \beta$-mercaptoethanol as described before ${ }^{31}$. Min6 cells were seeded in a six-well plate and allowed to attach overnight. Min 6 cells were incubated for $24 \mathrm{~h}$ before collection and analysis.

Islet isolation. Islets were isolated from 8 to 12 weeks old C57BL/6 male mice as described before by our laboratory ${ }^{31-34}$. Briefly, mouse islets were isolated using perfusion and digestion of pancreas with collagenase $\mathrm{V}$ (from Roche), density gradient purification with histopaque-1077 (Sigma), and then hand-picked. Isolated islets were cultured overnight in RPMI 1640 containing 10\% FBS, $11 \mathrm{mM}$ glucose, and then switched for $1 \mathrm{~h}$ to Krebs Ringer Bicarbonate buffer containing $2.6 \mathrm{mM} \mathrm{CaCl}_{2}, 1.2 \mathrm{mM} \mathrm{MgSO}_{4}, 1.2 \mathrm{mM} \mathrm{KH}_{2} \mathrm{PO}_{4}, 4.9 \mathrm{mM} \mathrm{KCl}, 98.5 \mathrm{mM}$ $\mathrm{NaCl}$, and $25.9 \mathrm{mM} \mathrm{NaHCO}_{3}$ (all from Sigma-Aldrich) supplemented with $20 \mathrm{mM} \mathrm{Na-HEPES}$ and $0.1 \%$ BSA. About 10 islets in each experimental condition were transferred to each well in 24-well plate containing $2.8 \mathrm{mM}$ and $16.7 \mathrm{mM}$ glucose concentration in Krebs Ringer Bicarbonate buffer for $1 \mathrm{~h}$. The supernatants were collected for insulin measurements. The islets were lysed with $1 \%$ Triton to determine total protein content in the islets. Insulin levels were measured with an ELISA kit from ALPCO. About 200 isolated mouse islets from WT or sparc $-/-$ mice were also collected for Western blot analysis of RGS4 and SPARC.

Western blot. Western blot analysis was performed using equal amounts of whole cell extract protein as described before ${ }^{33,35}$. Briefly, cell lysates were run on SDS PAGE, proteins transferred to a nitrocellulose membrane. The membranes were incubated with the primary antibodies, rabbit anti- RGS4 (Santa cruz), mouse antiSPARC (Haematologic Technologies, Inc), rabbit anti-AKT-S473, rabbit anti-AKT, mouse anti-beta-actin (Cell Signaling), respectively, followed by Horseradish peroxidase labeled donkey anti rabbit or donkey anti mouse antibodies. Protein signal was visualized by using Immun-Star chemiluminescent kit (Bio-Rad) and quantified by Bio-Rad Imager.

Adenoviral infection. Adenovirus was amplified in HEK-293 cells. The adenovirus in HEK-293 cells was collected and subjected to three cycles of freeze-thaw. Adenovirus titer was determined by using Adeno-X rapid titer kit from Clontech. Min6 cells or isolated islets were infected with AdV-SPARC or AdV-EGFP at 100 MOI for $16 \mathrm{~h}$. The next day, the medium was changed, and the cells were cultured for total $48 \mathrm{~h}$. We used AdV-EGFP virus as control and the expression of EGFP inside the core of islets was confirmed under fluorescent microscope.

siRNA transfection. Min6 cells were transfected with sparc siRNA or rgs4 siRNA or control scramble siRNA using Metafectene Pro, a cationic liposome-based transfection reagent (Biontex laboratories, Germany), which possessed high transfection efficiency in Min6 cells shown by the manufacturer. siRNA against mouse SPARC and mouse RGS4 were synthesized by Shanghai GenePharma Co. The sense sequence of mouse SPARC was: AGAAGCUGCGUGUGAAGAAUU. The sense sequence of mouse RGS4 was: CCUGCCUGAGGAGUCAAA TT.

$R T-q P C R$. RNA was first extracted using Trizol reagent (Invitrogen), then followed by purification and DNase I digestion to remove genomic DNA contamination using RNAeasy column (Qiagen) according to the manufacturer's protocol. After reverse transcription (Thermo Scientific), cDNA was quantified with ABI 7900HT Fast Real-Time PCR System (Applied Biosystems). Relative gene expression levels were calculated using the $\Delta \Delta \mathrm{Ct}$ method, with $18 \mathrm{~S}$ or GAPDH used as the reference gene and normalized. RGS4 qPCR primer sequences were: Sense: AGAAATGGGCTGAATCGTTG; Anti-sense: GGCTTGTCTCCTCTCTGGTG. 
A

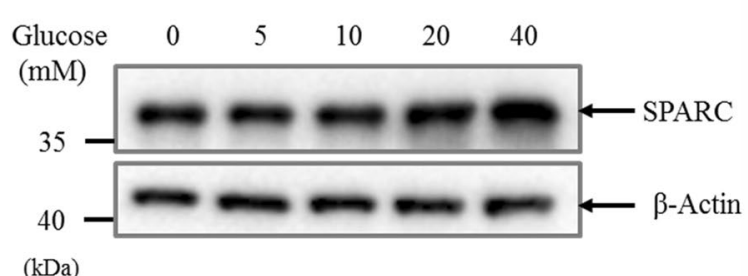

C

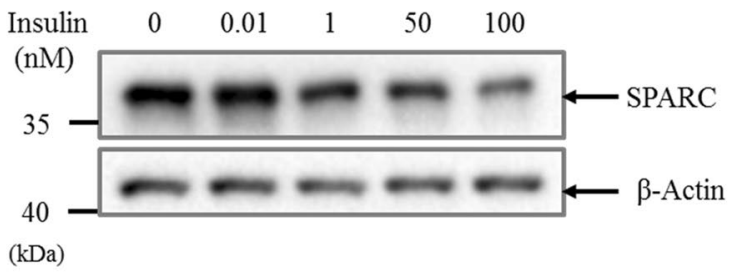

B

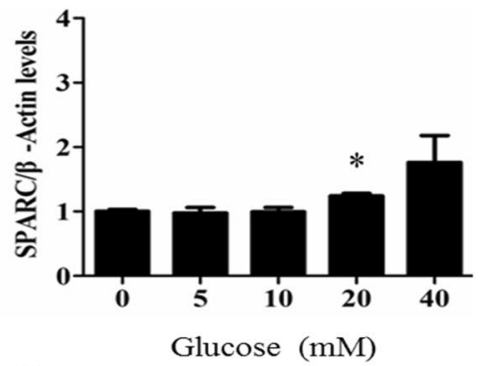

D

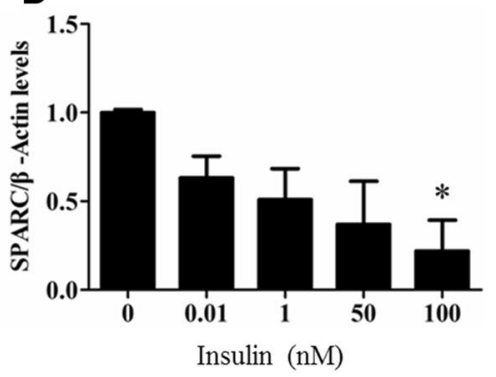

Figure 1. SPARC expression levels were regulated by glucose and insulin. (A) Min6 cells were incubated with increasing concentrations of glucose $(0-40 \mathrm{mM})$ for $16 \mathrm{~h}$. Cell lysates were subjected to western blotting with anti-SPARC antibody or $\beta$-actin antibody. (B) Min6 cells were stimulated with increasing concentration of insulin (0 to $100 \mathrm{nM}$ ) for $16 \mathrm{~h}$. Cell lysates were subjected to Western blotting with anti-SPARC antibody or $\beta$-actin antibody. (C,D) The SPARC levels were quantified relative to loading control $\beta$-actin each from three experiments. ${ }^{*}$ denotes $P<0.05, \mathrm{n}=3$. Two-way ANOVA followed by Tukey's multiple comparisons test was used in statistical analysis.

Microarray method. Min6 cells were infected with AdV-EGFP or AdV-SPARC for $48 \mathrm{~h}$. The cells were collected, and total RNA was prepared by Trizol and RNA easy column (Qiagen). The RNA samples were sent to Shanghai Biotechnology incorporation for customer cDNA microarray analysis using Affymetrix mouse array 4302.0 .

Statistics. Statistical significance was assessed by two-tailed Student $t$ test. For multiple groups, statistical significance was performed with one way or two-way ANOVA followed by Tukey's multiple comparisons test by GraphPad Prism 7. A value of $\mathrm{P}<0.05$ was considered significant. All data are expressed as the mean $\pm \mathrm{SE}$.

\section{Results}

Regulation of SPARC expression by glucose and insulin. To determine whether glucose and insulin regulate SPARC expression, we treated Min6 cells with increasing concentration of glucose or insulin. The results in Fig. 1A show that glucose concentration dose-dependently increased SPARC expression as shown by Western blot analysis. Conversely, insulin dose-dependently inhibited SPARC expression as assessed by Western blot analysis (Fig. 1C). Figure 1B,D are the results each from three experiments.

SPARC enhances insulin secretion in mouse pancreatic $\beta$ cells. To determine the effect of SPARC on insulin secretion, we overexpressed SPARC by adenovirus in isolated mouse islets. AdV-SPARC increased SPARC mRNA expression level by about 60-fold in isolated mouse islets, compared with control AdV-EGFP (Fig. S1A). SPARC overexpression enhanced muscarinic receptor agonist Oxotremorine M-stimulated insulin secretion (Fig. 2A). To confirm this effect, we also treated cells with recombinant SPARC protein. As shown in Fig. 2B, SPARC enhanced Oxotremorine M-stimulated insulin secretion in mouse islets. However, SPARC had no effect on insulin content (Fig. S1B). Meanwhile, we found that SPARC did not enhance KCl-stimulated insulin secretion in mouse islets (Fig. S1C). These results uncovered a specific role of SPARC in enhancement of Oxotremorine M-stimulated insulin secretion in mouse pancreatic $\beta$ cells.

Downregulation of RGS4 by Overexpression of SPARC. To study the changes of gene expression induced by overexpression of SPARC, we conducted cDNA microarray in Min6 cells overexpressing SPARC using Affimetrix mouse cDNA chip and found down-regulation by SPARC of RGS-4 mRNA to 2.6-folds compared to AdV-EGFP-infected Min6 cells (Fig. 3A); SPARC also induced Glut2 by twofold as shown in Fig. 3A. The detailed cDNA microarray data can be downloaded in the NCBI GEO site shown below: https://www.ncbi. nlm.nih.gov/geo/query/acc.cgi?acc=GSE155496.

Since RGS4 is a known negative regulator of insulin secretion through inhibition of muscarinic M3 receptor$\mathrm{G}(\mathrm{q})$ protein coupling in $\beta$ cells, we further investigated the effect of SPARC overexpression and loss of sparc 

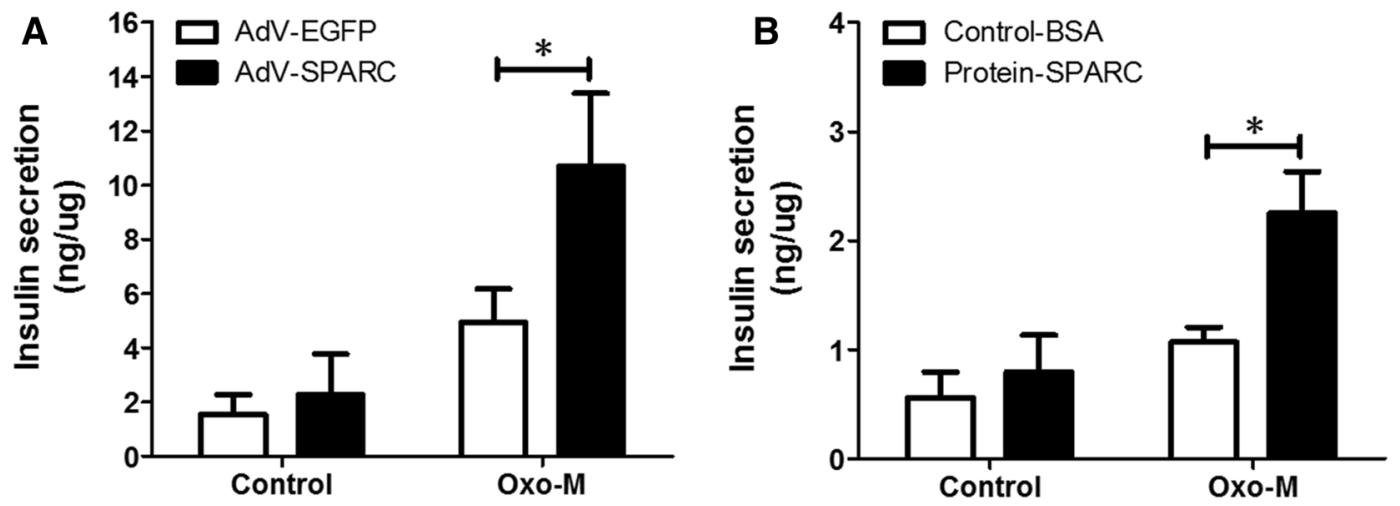

Figure 2. SPARC enhanced muscarinic receptor agonist-stimulated insulin secretion. (A) Mouse islets were isolated and culture in RPMI1640 containing $11 \mathrm{mM}$ glucose overnight. The islets were infected with AdVEGFP or AdV-SPARC for $24 \mathrm{~h}$. The islets were stimulated with $16.7 \mathrm{mM}$ glucose, or $16.7 \mathrm{mM}$ glucose and $100 \mu \mathrm{M}$ Oxo-M in Krebs Ringer Bicarbonate buffer for $1 \mathrm{~h}$. The supernatants were collected, and insulin was determined with insulin ELISA kit. Shown were the levels of insulin secretion normalized to protein concentration. The results were derived from three individual experiments. ${ }^{\star}$ denotes $P<0.05, \mathrm{n}=3$. (B) Mouse islets were cultured in RMPI1640 medium with $11 \mathrm{mM}$ glucose overnight. Groups of islets were then incubated with Krebs Ringer Bicarbonate buffer and with $16.7 \mathrm{mM}$ glucose in the presence or absence of recombinant SPARC, $1 \mu \mathrm{g} / \mathrm{ml}$, or $16.7 \mathrm{mM}$ glucose and $100 \mu \mathrm{M}$ Oxo-M in the presence or absence of SPARC for $1 \mathrm{~h}$. The supernatants were collected, and insulin was determined with insulin ELISA kit. Shown were the levels of insulin secretion normalized to protein concentration. ${ }^{\star}$ denotes $P<0.05, \mathrm{n}=3$. Statistical significance was assessed by unpaired two-tailed Student's t test.

on RGS4 expression. To confirm the microarray data, we overexpressed SPARC in Min6 cells and analyzed the mRNA expression levels on some of those genes shown in microarray heatmap (Fig. 3A) by RT-qPCR. As shown in Supplemental Fig. 2A, overexpression of SPARC in Min6 cells induced up-regulation of Glut2 mRNA expression. Glut 2 protein has been shown to be reduced in the islets from sparc-/- mice ${ }^{22}$. Meanwhile, although expression levels of several other $\beta$-cell genes were altered in microarray data, these changes were not confirmed by RT-qPCR experiment (Fig. S2A). We confirmed that overexpression of SPARC in Min6 cells induced downregulation of RGS4 mRNA (Fig. 3B) and protein (Fig. 3C,D).

Up-regulated RGS4 protein in pancreatic islets in sparc -/- mice. We reasoned that if SPARC inhibits RGS4 expression, knockdown of SPARC would increase RGS4 expression. As shown in Fig. 4A, treatment with siRNA against sparc gene transcripts reduced SPARC expression by $60 \%$ in Min6 cells (Fig. 4A), and the reduction of SPARC resulted in the increase in RGS4 expression by 2.5 -fold (Fig. 4A,B).

To further corroborate the siRNA result in Fig. 4A,B, we also examined the effect of loss of sparc gene on expression of RGS4 protein in the islet of sparc-/- mice. We isolated islets from wild type (WT) and sparc -I- mice, performed Western blot analysis and found that RGS4 protein levels were also up-regulated in the islets of sparc-/- mice (Fig. 4C,D). Consistent with the findings above, we found that up-regulation of RGS4 mRNA and down-regulation of Glut 2 mRNA expressions in the islet of sparc-/- mice compared to WT mice (Fig. S2B).

SPARC down-regulates RGS4 partially through activating Phosphoinositide 3-kinase and AKT signaling pathway. If SPARC down-regulates RGS4, we would expect SPARC to enhance insulin signaling. The results showed that overexpression of SPARC in Min6 cells induced AKT 473 phosphorylation (Fig. S3A,C), whereas knockdown of SPARC reduced AKT 473 phosphorylation (Fig. S3B,D).

To understand how SPARC might regulate RGS4 expression, we used LY294002, a selective inhibitor of PI3K, to treat Min 6 cells. Consistent with the results in Fig. 3C and Fig. S3A, the treatment of Min 6 cells with recombinant SPARC protein induced AKT 473 phosphorylation and down-regulation of RGS4 protein (Fig. 5A-C). LY-294002 at $10 \mu \mathrm{M}$ significantly decreased p-AKT (S473) expression levels and up-regulated RGS4 protein levels in Min6 cells (Fig. 5A-C). Furthermore, LY-294002 abolished induction of AKT 473 phosphorylation and of down-regulation of RGS4 protein by SPARC (Fig. 5A-C). Meanwhile, the changes of total AKT protein levels were not significant in either treatment. The data suggested that the activation of PI3K by SPARC played a role in down-regulation of RGS4 expression. Taken together, these results indicated that in Min6 cells, SPARC down-regulated RGS4 expression levels at least partially through activating PI3K signaling pathway.

Impaired insulin secretion in isolated islets of sparc -/- mice. To directly assess effect of SPARC on $\beta$-cell function, we carried out in vitro culture on isolated islets. First, to directly test the function of SPARC in $\beta$ cells, we measured insulin secretion under static incubation conditions by isolated islets obtained from WT and sparc-/- mice. As seen in Fig. 6A, the islets isolated from sparc-/- mice demonstrated significantly lower insulin secretion compared to those of WT mice in response to both high glucose and Oxo-M stimulation. Taken 
A

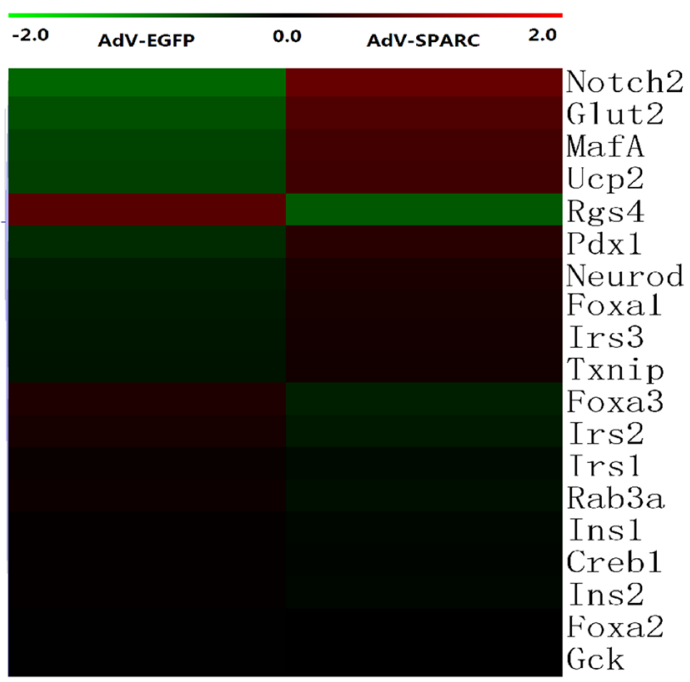

C

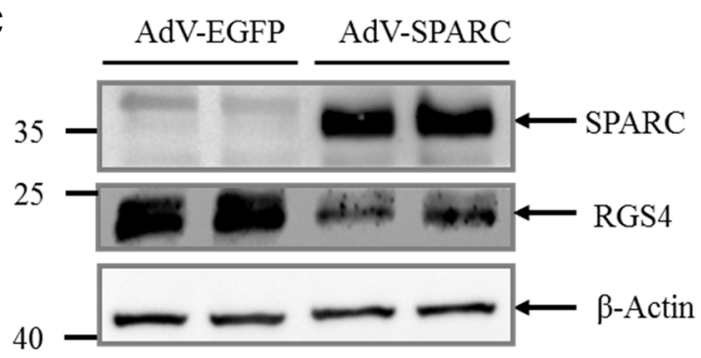

$(\mathrm{kDa})$
B

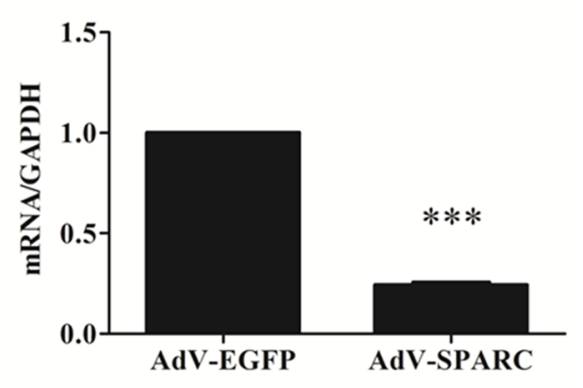

Figure 3. Overexpression of SPARC down-regulates RGS4 mRNA and protein in Min6 cells. Min6 cells were infected with AdV-EGFP or AdV-SPARC for $24 \mathrm{~h}$. (A) mRNAs were prepared from AdV-EGFP and AdV-SPACR treated Min6 cells and were subjected to cDNA microarray. The microarray data measuring relative expression of selected genes were shown in the heat map. (B) RGS4 mRNA was downregulated by overexpression of SPARC in Min6 cells. The cells were harvested for RNA preparation. RT-qPCR was performed to determine RGS4 mRNA levels after SPARC overexpression. (C) The cell lysates were subjected to Western blotting with anti-SPARC antibody or anti- $\beta$-actin antibody, and SPARC protein was shown to be overexpressed. The cell lysates were also subjected to Western blotting with anti-RGS4 antibody, and the results showed downregulation of RGS4 protein by overexpression of SPARC in Min 6 cells. (D) Relative RGS4 protein levels were quantified and normalized with $\beta$-actin. Statistical significance was assessed by unpaired two-tailed Student's $t$ test. ${ }^{* *}$ denotes $P<0.01, \mathrm{n}=3$. ${ }^{* * *}$ denotes $P<0.001, \mathrm{n}=3$.

together, these data indicated that SPARC was necessary for normal insulin secretory function in response to glucose and Oxo-M in vitro in mouse $\beta$ cells.

Inhibition of RGS4 in cultured islets from sparc -/- mice restores Oxo-M- stimulated GSIS. To verify that increased RGS4 in sparc-/- mice is linked to impaired GSIS, we inhibited RGS4 function by administering CCG4986, a specific RGS4 inhibitor, in cultured islets isolated from sparc -/- mice. In islets of sparc -/mice, both $16.7 \mathrm{mM}$ high glucose and high glucose plus Oxo-M agonist did not induce GSIS (Fig. 6A). However, RGS4 inhibitor CCG4986 restored high glucose and Oxo-M-induced GSIS (Fig. 6B). CCG4986 increased insulin secretion by threefold, compared with control DMSO under high glucose plus Oxo-M condition. Furthermore, in order to confirm that SPARC regulates insulin secretion primarily through RGS4, we used siRNA against rgs4 gene transcripts to knockdown RGS4 expression. As shown in Fig. 6C, treatment with siRNA reduced RGS4 expression by $80 \%$ in Min6 cells (Fig. 6C), and knockdown of RGS4 partially decreased the promoting effect of SPARC on oxotremorine-M stimulated insulin secretion (Fig. 6D). However, knockdown of RSG4 had no effect on KCl-stimulated insulin secretion (Fig. S4A). Taken together, these results suggested a potential pathway by which SPARC regulated insulin secretion through RGS4 (Fig. 6E).

\section{Discussion}

In this study, we found that overexpression of SPARC inhibited RGS4 expression in Min6 cells; conversely, absence of SPARC increased RGS4 expression in the islets of sparc -/- mice. SPARC overexpression or exogenously administered recombinant SPARC increased muscarinic receptor agonist-stimulated insulin secretion. Here, we described a mechanism for regulating $\beta$-cell function through SPARC-RGS4 pathway (Fig. 6E). The 
A

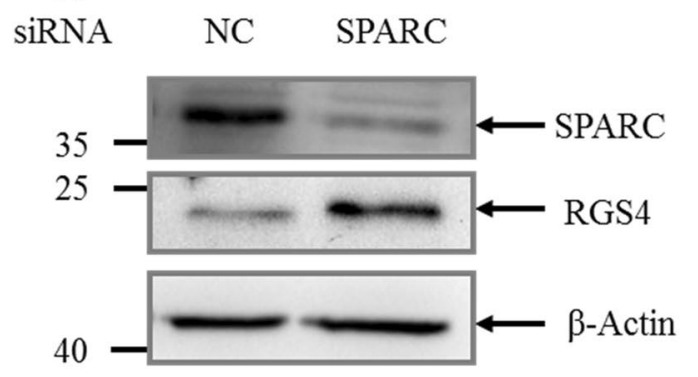

$(\mathrm{kDa})$

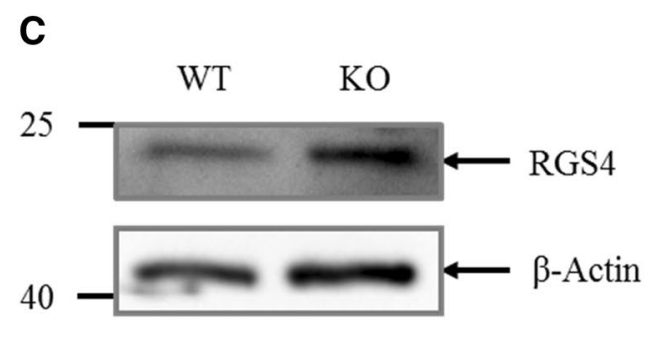

B
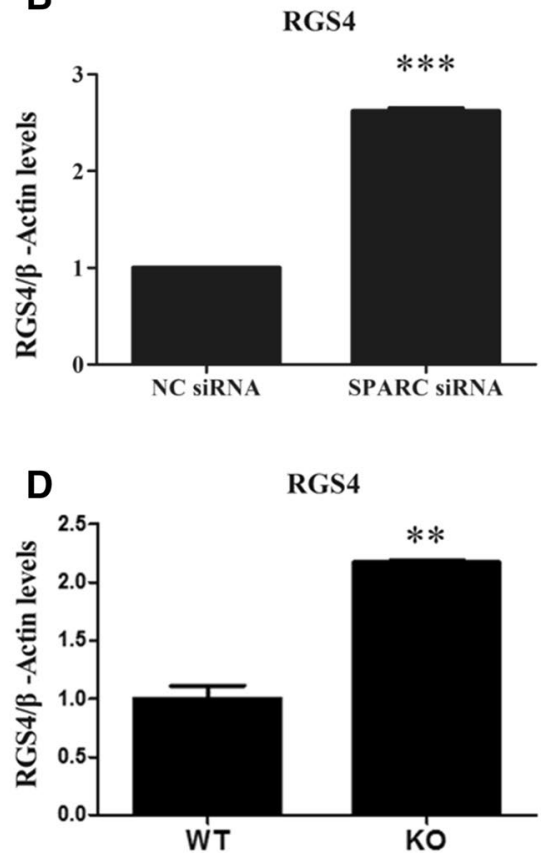

Figure 4. Knocking down of SPARC with siRNA in Min6 cells up-regulated RGS4 mRNA, and loss of sparc up-regulated RGS4 protein in isolated islets. Min6 cells were transfected with SPARC siRNA and cultured for $48 \mathrm{~h}$. (A,B) The cell lysates were subjected to Western blotting with anti-SPARC antibody or anti- $\beta$-actin antibody (A). (B) Relative RGS4 levels were quantified and normalized with $\beta$-actin. ${ }^{* * *}$ denotes $P<0.001, \mathrm{n}=3$. Statistical significance was assessed by unpaired two-tailed Student's t test. (C,D) Up-regulation of RGS4 protein in the islets of sparc-/- mice. Mouse islets were isolated from WT or sparc-/- mice and subject to Western blot analysis of RGS4 protein (C). Average RGS4 levels from two experiments were quantified and normalized to $\beta$-actin levels (D). ${ }^{* *}$ denotes $\mathrm{P}<0.01, \mathrm{n}=3$.

impaired insulin secretion represents a primary $\beta$-cell defect because isolated islets from sparc-/- mice exhibited impaired GSIS compared to WT islets.

Harries et al. previously showed that SPARC is mostly expressed and secreted by adipose tissue and SPARC increases human islet insulin secretion ${ }^{36}$. Therefore, increased plasma SPARC in patients with obesity would play a role in induction of insulin secretion. Stable SPARC-expressing rat INS cell line also increases high glucosestimulated insulin secretion, but the mechanism was not clear ${ }^{36}$. Our study in Min 6 cell line and in isolated mouse islets showed that SPARC increased muscarinic receptor agonist-stimulated insulin secretion, suggesting that one of the mechanisms by which SPARC increases insulin secretion may be through suppression of RGS4 expression. RGS4 is one of seven members of the classic R4 RGS protein family that accelerate the intrinsic GTPase activity of Gai/o and Gaq/11 family members. RGS4 inhibits the strength and duration of Gq signaling. In $\beta$-cells, RGS4 has been shown to negatively regulate insulin secretion through regulation of M3 receptor-G protein coupling ${ }^{26}$. During meals, acetylcholine is released from intra-pancreatic parasympathetic nerve endings and bound to M3 receptors on $\beta$ cells, resulting in activation of PLC $\gamma$, IP3 and calcium release, and insulin secretion. We showed that increased SPARC expression inhibited RGS4 expression, which, in turn, would result in increased M3 receptor G-protein coupling and increased insulin secretion (Fig. 6E).

Our results were consistent with a recent study demonstrating that SPARC is necessary for insulin secretion in mouse $\beta$ cells and that sparc-/- mice displayed glucose intolerance and diabetes in an age-dependent manner ${ }^{22}$. Moreover, Glucose transporter 2 (Glut2) expression in $\beta$ cells is also reduced in sparc $-/-$ mice $^{22}$, which was in agreement with our cDNA microarray and RT-qPCR data showing induction of Glut 2 by SPARC in Min 6 cells (Fig. 3A and Fig. S2A). In mouse $\beta$ cells, receptor-activated Gq leads to the sequential activation of ERK1/2 and IRS2 signaling and selective increases in the expression of islet genes involved in insulin synthesis (Ins2, Pcks1, and Pcks2), and Glut2, thus improving $\beta$-cell function ${ }^{37}$. In Rat INS1-M3 insulinoma cell line, M3 receptor-mediated activation of Gq leads to increased Irs2 expression via sequential activation of PLC (PLC $\beta$ ), PKC, and ERK1/2 $2^{37,38}$.

PI3K activation inhibits RGS4 mRNA and protein in rabbit colonic smooth muscle cells, which is reversed by PI3K inhibitor LY-294002 ${ }^{39}$. We hypothesized that SPARC down-regulated RGS-4 expression through PI3K activation. Treatment of Min6 cells with LY-294002 blocked SPARC-induced down-regulation of RGS4 protein (Fig. 5A), demonstrating that PI3K pathway regulated RGS4 downregulation induced by SPARC in Min6 cells. Our study showed that SPARC increased AKT phosphorylation in Min6 cells (Fig. S3A), which is downstream of PI3K. Akt phosphorylation has also contributed to increased insulin secretion ${ }^{40}$. SPARC also activates the 


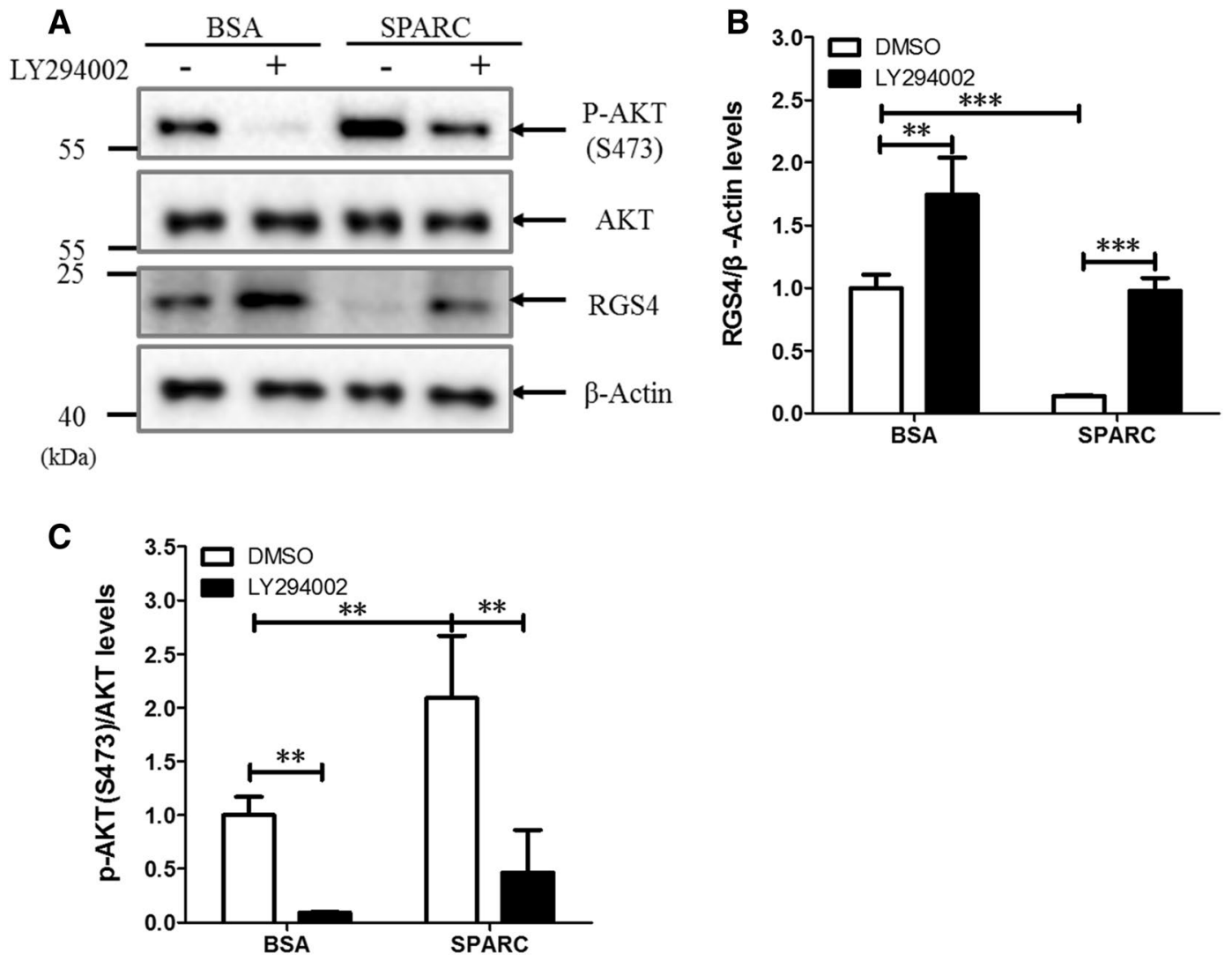

Figure 5. SPARC-induced AKT phosphorylation and RGS4 down-regulation in Min6 cells is abolished by PI3 kinase inhibitor LY-294002. (A) SPARC-induced RGS4 downregulation was blocked by PI3K inhibitor. Cultured Min6 cells after serum starvation for $12 \mathrm{~h}$ were pretreated with the PI3K inhibitor LY-294002 $(10 \mu \mathrm{M})$ for $1 \mathrm{~h}$ before treatment with or without recombinant SPARC $(1 \mu \mathrm{g} / \mathrm{ml})$ for $12 \mathrm{~h}$ followed by Western blot analysis with the indicated specific antibodies. Down-regulation of RGS4 by SPARC protein was abolished by LY-294002 (B). Lower panel was ratio of p-AKT S473/total AKT quantified from three experiments (C). ${ }^{* *}$ denotes $P<0.01$, $\mathrm{n}=3$. ${ }^{\star \star \star}$ denotes $P<0.001, \mathrm{n}=3$. Statistical significance was assessed by unpaired two-tailed Student's t test.

PI3K/AKT pathway to promote proliferation and metastasis in oral squamous cell carcinoma ${ }^{41}$, suggesting this pathway to be ubiquitous.

Our study suggested that it may be possible to use RGS4 inhibitors to increase insulin secretion. There are several RGS4 inhibitors that would be of interest to test if they can be used to increase insulin secretion. CCG4986 inhibits RGS4 function through the covalent modification of two spatially distinct cysteine residues on RGS4 ${ }^{42}$. Our in vitro GSIS experiments showed that CCG4986 restored GSIS in the isolated islets of sparc-/- mice, suggesting that the potential mechanism of defective insulin secretion was due to upregulated RGS4.

RGS4 dysfunction has been linked to many diseases, including hypertension, schizophrenia, and Parkinson's disease $^{43-45}$. RGS4 is induced by IL- $1 \beta$ through activation of NF- $\mathrm{KB}$, and RGS4 induction can in turn cause $\beta$-cell dysfunction by inhibiting insulin secretory machinery as exemplified before ${ }^{46}$. Our results suggested that increased RGS4 expression in sparc - / $\beta$ cells was mechanistically linked to a more chronic state of $\beta$-cell dysfunction. Since blood SPARC and insulin levels are increased in obesity and early type 2 diabetes ${ }^{47}$, our results also suggested the involvement of SPARC in islet adaptation to insulin resistance in humans by increasing muscarinic receptor-mediated insulin secretion. However, whether SPARC also downregulates RGS4 expression in human $\beta$-cells remains to be confirmed. Furthermore, SPARC receptor in $\beta$-cells remains to be identified.

In conclusion, we had identified a novel mechanism for SPARC to regulate insulin secretion by down-regulation of RGS4 expression and showed that SPARC played a role in the physiology of insulin secretion and in G-protein signaling in $\beta$ cells. In the future, it will be interesting to learn whether SPARC can improve insulin secretion in patients with type 2 diabetes through RGS4 pathway. 
A

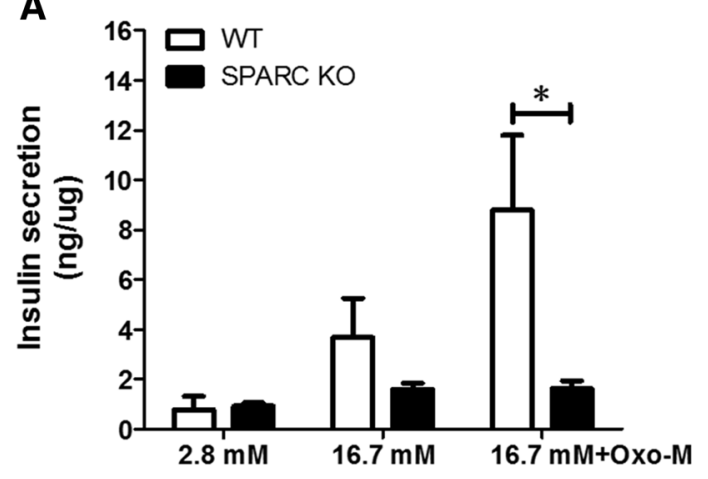

C

siRNA NC RGS4

25

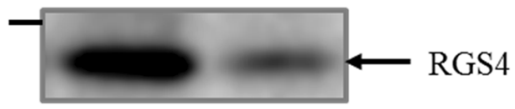

40

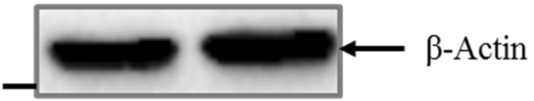

B

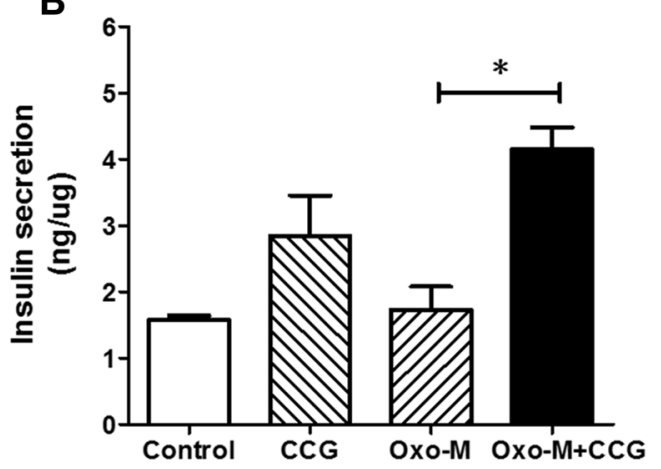

$(\mathrm{kDa})$

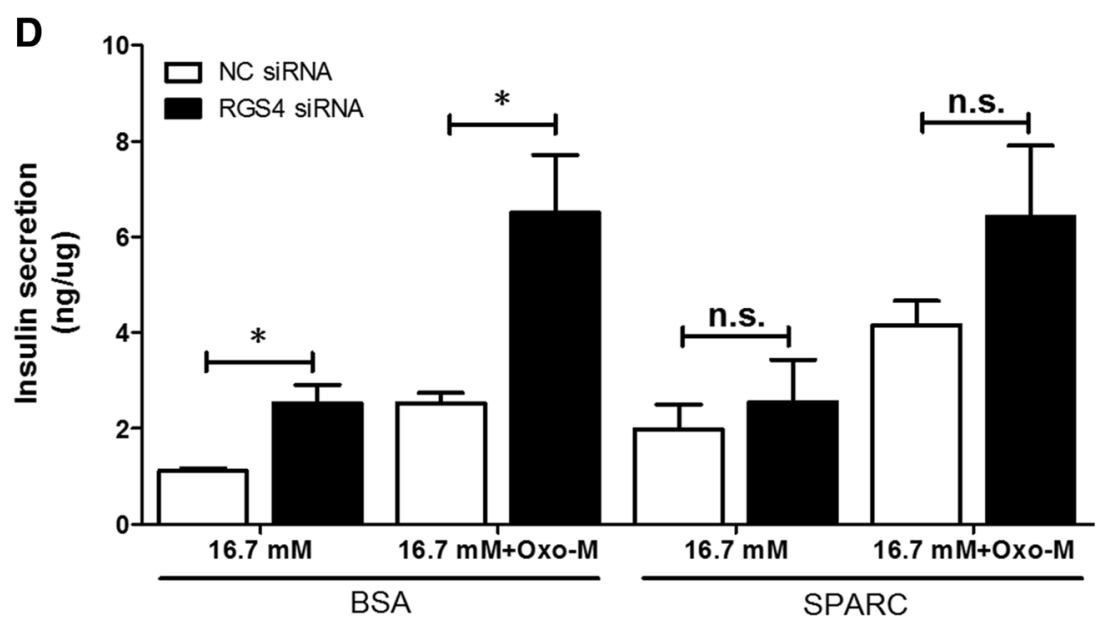

E

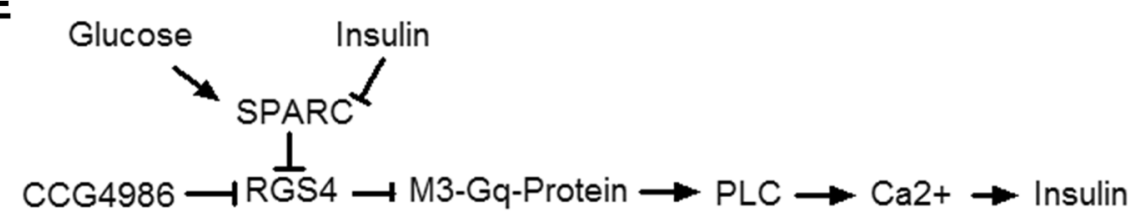

Figure 6. RGS4 inhibitor CCG4986 restored insulin secretion in islets of sparc-/- mice. (A) Mouse islets were isolated from WT or sparc $-/-$ mice. The isolated islets were cultured for $16 \mathrm{~h}$ in RPMI1640 with $11 \mathrm{mM}$ Glucose and 10\% FBS. The WT or sparc-/- islets were incubated for $1 \mathrm{~h}$ in $2.8 \mathrm{mM}$ glucose, then the medium was changed to $16.7 \mathrm{mM}$ glucose, $16.7 \mathrm{mM}$ glucose with Oxo-M, $100 \mu \mathrm{M}$ in Krebs Ringer Bicarbonate buffer for $1 \mathrm{~h}$. The supernatants were collected for insulin assay. Shown were the levels of insulin secretion normalized to total protein concentrations. ${ }^{*}$ denotes $P<0.05, \mathrm{n}=3$. Statistical significance was assessed by unpaired two-tailed Student's t test. (B) sparc-/- mouse islets were incubated for $1 \mathrm{~h}$ in $2.8 \mathrm{mM}$ glucose, and then the medium was changed to $16.7 \mathrm{mM}$ glucose, $16.7 \mathrm{mM}$ glucose with Oxo-M, $100 \mu \mathrm{M}$, or $16.7 \mathrm{mM}$ glucose + CCG4986, $100 \mu \mathrm{M}$, or $16.7 \mathrm{mM}$ glucose, Oxo-M, $100 \mu \mathrm{M}$, CCG4986, $100 \mu \mathrm{M}$. The supernatants were collected, and insulin was measured. Shown were the levels of insulin secretion normalized to protein concentration. ${ }^{*}$ denotes $P<0.05$, $\mathrm{n}=3$. Two-way ANOVA followed by Tukey's multiple comparisons test was used in statistical analysis. (C,D) Min6 cells were transfected with RGS4 siRNA and cultured for $48 \mathrm{~h}$. The cell lysates were subjected to Western blotting with anti-RGS4 antibody or anti- $\beta$-actin antibody (C). (D) After being transfected with RGS4 siRNA for $48 \mathrm{~h}$, groups of Min 6 cells were then incubated with $16.7 \mathrm{mM}$ glucose, or $16.7 \mathrm{mM}$ glucose and $100 \mu \mathrm{M}$ Oxo-M in the presence or absence of recombinant SPARC, $1 \mu \mathrm{g} / \mathrm{ml}$ for $1 \mathrm{~h}$. The supernatants were collected, and insulin was measured. Shown were the levels of insulin secretion normalized to protein concentration. n.s. denotes $P>0.05, \mathrm{n}=3$. $^{*}$ denotes $P<0.05, \mathrm{n}=3$. Statistical significance was assessed by unpaired two-tailed Student's $t$ test. (E) The graph schematically outlined a potential novel mechanism for the regulation of insulin secretion by SPARC. Increased SPARC inhibited RGS4 expression, which, in turn, would result in increased M3 receptor / G-protein coupling, leading to increased insulin secretion in $\beta$ cells. 
Received: 24 December 2019; Accepted: 5 October 2020

Published online: 16 October 2020

\section{References}

1. Defronzo, R. A. Banting Lecture. From the triumvirate to the ominous octet: A new paradigm for the treatment of type 2 diabetes mellitus. Diabetes 58, 773-795. https://doi.org/10.2337/db09-9028 (2009).

2. Olefsky, J. M. \& Glass, C. K. Macrophages, inflammation, and insulin resistance. Annu. Rev. Physiol. 72, 219-246. https://doi. org/10.1146/annurev-physiol-021909-135846 (2010).

3. Weir, G. C. \& Bonner-Weir, S. Five stages of evolving beta-cell dysfunction during progression to diabetes. Diabetes 53(Suppl 3), S16-21 (2004).

4. Kwon, H. \& Pessin, J. E. Adipokines mediate inflammation and insulin resistance. Front. Endocrinol. 4, 71. https://doi.org/10.3389/ fendo.2013.00071 (2013).

5. Lane, T. F. \& Sage, E. H. The biology of SPARC, a protein that modulates cell-matrix interactions. FASEB J. 8, 163-173 (1994)

6. Kucukdereli, H. et al. Control of excitatory CNS synaptogenesis by astrocyte-secreted proteins Hevin and SPARC. Proc. Natl. Acad. Sci. U.S.A. 108, E440-449. https://doi.org/10.1073/pnas.1104977108 (2011).

7. Bradshaw, A. D. Diverse biological functions of the SPARC family of proteins. Int. J. Biochem. Cell Biol. 44, 480-488. https://doi. org/10.1016/j.biocel.2011.12.021 (2012).

8. Rivera, L. B. \& Brekken, R. A. SPARC promotes pericyte recruitment via inhibition of endoglin-dependent TGF-betal activity. J. Cell Biol. 193, 1305-1319. https://doi.org/10.1083/jcb.201011143 (2011).

9. Bradshaw, A. D., Graves, D. C., Motamed, K. \& Sage, E. H. SPARC-null mice exhibit increased adiposity without significant differences in overall body weight. Proc. Natl. Acad. Sci. U.S.A. 100, 6045-6050. https://doi.org/10.1073/pnas.1030790100 (2003).

10. Cheng, L., Sage, E. H. \& Yan, Q. SPARC fusion protein induces cellular adhesive signaling. PLoS ONE 8, e53202. https://doi. org/10.1371/journal.pone.0053202 (2013).

11. Barker, T. H. et al. SPARC regulates extracellular matrix organization through its modulation of integrin-linked kinase activity. J. Biol. Chem. 280, 36483-36493. https://doi.org/10.1074/jbc.M504663200 (2005).

12. Kos, K. \& Wilding, J. P. SPARC: a key player in the pathologies associated with obesity and diabetes. Nat. Rev. Endocrinol. 6, 225-235. https://doi.org/10.1038/nrendo.2010.18 (2010).

13. Kos, K. et al. Regulation of the fibrosis and angiogenesis promoter SPARC/osteonectin in human adipose tissue by weight change, leptin, insulin, and glucose. Diabetes 58, 1780-1788. https://doi.org/10.2337/db09-0211 (2009).

14. Nie, J. \& Sage, E. H. SPARC functions as an inhibitor of adipogenesis. J. Cell Commun. Signal. 3, 247-254. https://doi.org/10.1007/ s12079-009-0064-4 (2009).

15. Nie, J. \& Sage, E. H. SPARC inhibits adipogenesis by its enhancement of beta-catenin signaling. J. Biol. Chem. 284, 1279-1290. https://doi.org/10.1074/jbc.M808285200 (2009).

16. Shen, Y. et al. SPARC is over-expressed in adipose tissues of diet-induced obese rats and causes insulin resistance in 3T3-L1 adipocytes. Acta Histochem. 116, 158-166. https://doi.org/10.1016/j.acthis.2013.06.004 (2014).

17. Okura, Y. et al. Interaction of nerve growth factor beta with adiponectin and SPARC oppositely modulates its biological activity. Int. J. Mol. Sci. https://doi.org/10.3390/ijms20071541 (2019).

18. Song, H., Ding, L., Zhang, S. \& Wang, W. MiR-29 family members interact with SPARC to regulate glucose metabolism. Biochem. Biophys. Res. Commun. 497, 667-674. https://doi.org/10.1016/j.bbrc.2018.02.129 (2018).

19. Song, H., Guan, Y., Zhang, L., Li, K. \& Dong, C. SPARC interacts with AMPK and regulates GLUT4 expression. Biochem. Biophys. Res. Commun. 396, 961-966. https://doi.org/10.1016/j.bbrc.2010.05.033 (2010).

20. Aseer, K. R., Silvester, A. J., Kumar, A., Choi, M. S. \& Yun, J. W. SPARC paucity alleviates superoxide-mediated oxidative stress, apoptosis, and autophagy in diabetogenic hepatocytes. Free Radical Biol. Med. 108, 874-895. https://doi.org/10.1016/j.freeradbio med.2017.05.011 (2017).

21. Ryall, C. L. et al. Novel role for matricellular proteins in the regulation of islet beta cell survival: The effect of SPARC on survival, proliferation, and signaling. J. Biol. Chem. 289, 30614-30624. https://doi.org/10.1074/jbc.M114.573980 (2014).

22. Atorrasagasti, C. et al. SPARC is required for the maintenance of glucose homeostasis and insulin secretion in mice. Clin. Sci. 133, 351-365. https://doi.org/10.1042/CS20180714 (2019).

23. Nie, J., Bradshaw, A. D., Delany, A. M. \& Sage, E. H. Inactivation of SPARC enhances high-fat diet-induced obesity in mice. Connect. Tissue Res. 52, 99-108. https://doi.org/10.3109/03008207.2010.483747 (2011).

24. Gautam, D. et al. Role of the M3 muscarinic acetylcholine receptor in beta-cell function and glucose homeostasis. Diabetes Obes. Metab. 9(Suppl 2), 158-169. https://doi.org/10.1111/j.1463-1326.2007.00781.x (2007).

25. Ruiz de Azua, I., Gautam, D., Jain, S., Guettier, J. M. \& Wess, J. Critical metabolic roles of beta-cell M3 muscarinic acetylcholine receptors. Life Sci. 91, 986-991. https://doi.org/10.1016/j.lfs.2012.04.010 (2012).

26. Ruiz de Azua, I. et al. RGS4 is a negative regulator of insulin release from pancreatic beta-cells in vitro and in vivo. Proc. Natl. Acad. Sci. USA 107, 7999-8004. https://doi.org/10.1073/pnas.1003655107 (2010).

27. Said, N., Frierson, H. F., Sanchez-Carbayo, M., Brekken, R. A. \& Theodorescu, D. Loss of SPARC in bladder cancer enhances carcinogenesis and progression. J. Clin. Investig. 123, 751-766. https://doi.org/10.1172/JCI64782 (2013).

28. Luo, Z. et al. SPARC deficiency affects bone marrow stromal function, resulting in impaired B lymphopoiesis. J. Leukoc. Biol. https ://doi.org/10.1189/jlb.1A0713-415RR (2014).

29. Socha, M. J. et al. Aberrant promoter methylation of SPARC in ovarian cancer. Neoplasia 11, 126-135 (2009).

30. Dai, Y. S., Xu, J. \& Molkentin, J. D. The DnaJ-related factor Mrj interacts with nuclear factor of activated T cells c3 and mediates transcriptional repression through class II histone deacetylase recruitment. Mol. Cell. Biol. 25, 9936-9948. https://doi.org/10.1128/ MCB.25.22.9936-9948.2005 (2005).

31. Luo, Y. et al. Transcription factor Ets1 regulates expression of thioredoxin-interacting protein and inhibits insulin secretion in pancreatic beta-cells. PLoS ONE 9, e99049. https://doi.org/10.1371/journal.pone.0099049 (2014).

32. Zhang, J. et al. Disruption of growth factor receptor-binding protein 10 in the pancreas enhances beta-cell proliferation and protects mice from streptozotocin-induced beta-cell apoptosis. Diabetes 61, 3189-3198. https://doi.org/10.2337/db12-0249 (2012).

33. Hu, L. et al. NFATc3 deficiency reduces the classical activation of adipose tissue macrophages. J. Mol. Endocrinol. 61, 79-89. https ://doi.org/10.1530/JME-18-0070 (2018).

34. Hu, L. et al. Reduced compensatory beta-cell proliferation in Nfatc3-deficient mice fed on high-fat diet. Exp. Clin. Endocrinol. Diabetes https://doi.org/10.1055/a-1008-9110 (2019).

35. Dai, Y. S., Cserjesi, P., Markham, B. E. \& Molkentin, J. D. The transcription factors GATA4 and dHAND physically interact to synergistically activate cardiac gene expression through a p300-dependent mechanism. J. Biol. Chem. 277, 24390-24398. https:// doi.org/10.1074/jbc.M202490200 (2002).

36. Harries, L. W. et al. A role for SPARC in the moderation of human insulin secretion. PLoS ONE 8, e68253. https://doi.org/10.1371/ journal.pone.0068253 (2013).

37. Jain, S. et al. Chronic activation of a designer G(q)-coupled receptor improves beta cell function. J. Clin. Investig. 123, 1750-1762. https://doi.org/10.1172/JCI66432 (2013). 
38. Iismaa, T. P. et al. Quantitative and functional characterization of muscarinic receptor subtypes in insulin-secreting cell lines and rat pancreatic islets. Diabetes 49, 392-398 (2000).

39. Hu, W., Li, F., Mahavadi, S. \& Murthy, K. S. Interleukin-1beta up-regulates RGS4 through the canonical IKK2/IkappaBalpha/ NF-kappaB pathway in rabbit colonic smooth muscle. Biochem. J. 412, 35-43. https://doi.org/10.1042/BJ20080042 (2008).

40. Bernal-Mizrachi, E. et al. Defective insulin secretion and increased susceptibility to experimental diabetes are induced by reduced Akt activity in pancreatic islet beta cells. J. Clin. Investig. 114, 928-936. https://doi.org/10.1172/JCI20016 (2004).

41. Jing, Y. et al. SPARC promotes the proliferation and metastasis of oral squamous cell carcinoma by PI3K/AKT/PDGFB/PDGFRbeta axis. J. Cell Physiol. https://doi.org/10.1002/jcp.28205 (2019).

42. Kimple, A. J. et al. The RGS protein inhibitor CCG-4986 is a covalent modifier of the RGS4 Galpha-interaction face. Biochem. Biophys. Acta. 1774, 1213-1220. https://doi.org/10.1016/j.bbapap.2007.06.002 (2007).

43. Kattoulas, E. et al. Schizophrenia-related RGS4 gene variations specifically disrupt prefrontal control of saccadic eye movements. Psychol. Med. 42, 757-767. https://doi.org/10.1017/S003329171100167X (2012).

44. Gu, S., Cifelli, C., Wang, S. \& Heximer, S. P. RGS proteins: Identifying new GAPs in the understanding of blood pressure regulation and cardiovascular function. Clin. Sci. 116, 391-399. https://doi.org/10.1042/CS20080272 (2009).

45. Lerner, T. N. \& Kreitzer, A. C. RGS4 is required for dopaminergic control of striatal LTD and susceptibility to parkinsonian motor deficits. Neuron 73, 347-359. https://doi.org/10.1016/j.neuron.2011.11.015 (2012).

46. Hu, W., Li, F., Mahavadi, S. \& Murthy, K. S. Upregulation of RGS4 expression by IL-1beta in colonic smooth muscle is enhanced by ERK1/2 and p38 MAPK and inhibited by the PI3K/Akt/GSK3beta pathway. Am. J. Physiol. Cell Physiol. 296, C1310-1320. https ://doi.org/10.1152/ajpcell.00573.2008 (2009).

47. Wu, D., Li, L., Yang, M., Liu, H. \& Yang, G. Elevated plasma levels of SPARC in patients with newly diagnosed type 2 diabetes mellitus. Eur. J. Endocrinol. Eur. Feder. Endocr. Soc. 165, 597-601. https://doi.org/10.1530/EJE-11-0131 (2011).

\section{Acknowledgements}

This work is supported by Natural Science Foundation of China Grant no. 81270905 (Y-S Dai).

\section{Author contributions}

L.H., F.H., M.H. researched data. Q.Z., L.C., N.S. contributed materials. F.L., Z.Z. contributed to discussion, reviewed manuscript. L.H., Y.-S.D. researched data and wrote manuscript.

\section{Competing interests}

Y-S.D. is currently employed by Bristol-Myers Squibb Company. The rest of authors declare that there is no conflict of interest that could be perceived as prejudicing the impartiality of the research reported.

\section{Additional information}

Supplementary information is available for this paper at https://doi.org/10.1038/s41598-020-74593-w.

Correspondence and requests for materials should be addressed to Y.-S.D.

Reprints and permissions information is available at www.nature.com/reprints.

Publisher's note Springer Nature remains neutral with regard to jurisdictional claims in published maps and institutional affiliations.

(c)

Open Access This article is licensed under a Creative Commons Attribution 4.0 International License, which permits use, sharing, adaptation, distribution and reproduction in any medium or format, as long as you give appropriate credit to the original author(s) and the source, provide a link to the Creative Commons licence, and indicate if changes were made. The images or other third party material in this article are included in the article's Creative Commons licence, unless indicated otherwise in a credit line to the material. If material is not included in the article's Creative Commons licence and your intended use is not permitted by statutory regulation or exceeds the permitted use, you will need to obtain permission directly from the copyright holder. To view a copy of this licence, visit http://creativecommons.org/licenses/by/4.0/.

(C) The Author(s) 2020 